\title{
Spontaneous angina bullosa hemorrhagica of tongue: An unusual problem following prosthodontic procedure
}

\author{
Suresh K. Sachdeva*, Ashwani Sachdeva**, Swati Saggar Sachdeva***, Ashok Galav**** \\ *Department of Oral Medicine and Radiology, Surendera Dental College and Research Institute, India \\ **Department of Prosthodontics, JCD Dental College \& Hospital, India \\ ***Department of Prosthodontics, Eklavya Dental College \& Hospital, India \\ ****Department of Oral Medicine and Radiology, Tatya Saheb Kore Dental College \& Research \\ Centre, India
}

\begin{abstract}
Introduction: Angina bullosa hemorrhagica $(\mathrm{ABH})$ is the term used to describe benign subepithelial oral mucosal blood filled blisters, which are not attributed to any systemic disorder. It is a very uncommon condition, mostly seen in elderly patients. The exact etiopathogenesis of the ABH is not known. Herewith, we present a case of 50 -year-old male patient who developed $\mathrm{ABH}$ on right lateral border of the tongue, following prosthodontics impression making for completely edentulous mouth. Methods: The case management method was topical application of chlorhexidine gel $(1 \%)$ and lignocaine gel $(2 \%)$ with avoidance of hot and spicy food. Result: After one week in treating with topical therapy, the lesion became normal. The diagnosis of $A B H$ is difficult in patients because of its asymptomatic nature. The early diagnosis of the lesion is very important as a rapidly expanding blood-filled bulla in the oropharynx can cause upper airway obstruction. Therefore, a high level of suspicion is warranted on part of dentists who may the first to encounter the lesion. Conclusion: Spontaneous Angina Bullosa Hemorrhagica of tongue was an unusual problem that can occur following prosthodontic procedure. The management of the lession were intended to reduce pain or discomfort and prevent secondary infections.
\end{abstract}

Keywords: Angina bullosa hemorrhagica, prosthodontic impression, tongue

P-ISSN 1979-0201, e-ISSN 2549-6212 Available from:http://jurnal.unpad.ac.id/pjd/index

DOI:http://dx.doi.org/10.24198/pjd.vol29no2.12366

Submission: May 2017 Publishing: July 2017

\section{INTRODUCTION}

The word Angina bullosa hemorrhagica $(\mathrm{ABH})$ has been taken from the Latin verb "angere", which means to choke on. It is a rare disorder of unknown etiology and was first described and characterized by Badham ${ }^{1}$ as the sudden onset of blisters in the oral and oropharyngeal mucosa that cannot be attributed to any vesiculobullous disorders, blood dyscrasias, autoimmune

Corresponding author: Dr. Suresh K. Sachdeva, 13/573, Gali No. 1, Khanna Colony, Sirsa-125055 Haryana, India Email: drsureshsachdeva7184@gmail.com 
conditions or vascular disease. In 1933, Balina² of Argentina had already described the same lesions under the term traumatic oral hemophlyctenosis (TOH). This entity was later renamed as recurrent oral hemophlyctenosis (ROH). According to Kirtschig and Happle ${ }^{3}$, the term ABH is misleading as the most bullae arising in the oral cavity are not consistent with lesions usually called "angina"; subsequently they proposed a new term, stomatopompholyx hemorrhagica for this condition.

The blisters formed in $\mathrm{ABH}$ are dark red to purple in colour, solitary or multiple and may cause some discomfort. ${ }^{4}$ However, in general they are asymptomatic and are only observed incidentally or when their content is spilled out in the oral cavity. Mostly ABH affect the soft palate, but can also occur in the anterior pillar of the fauces, epiglottis, arytenoids, pharyngeal wall, and esophagus. $\mathrm{ABH}$ is an acute disease which is generally associated with trauma, and can present with frequent recurrences. ${ }^{5}$ Herewith, we presented a case of 50-year-old male patient who developed $\mathrm{ABH}$ on the right lateral border of the tongue, following prosthodontics impression procedure for complete denture fabrication.

A 50-year-old male patient was referred to the Department of Oral Medicine \& Radiology from the Department of Prosthodontics with a complaint of blood filled blister on the right lateral border of tongue. History of presenting illness revealed that patient was completely edentulous and had reported for complete denture fabrication to the department of prosthodontics. During primary impression making with impression materials, due to overheated impression tray, there was sudden appearance of blood filled blister on the right lateral border of the tongue.

There was no pain or any burning sensation. The patient's past medical history was not significant for any blood dyscrasias, anticoagulant therapy, or liver disease. Past dental history included extraction of all teeth due to periodontal problem, few years back. Patient was known smoker with 5 bidis/day from past 20 years. On general physical examination, patient was moderately built, well oriented to time, place and person with no skin, or eye lesions. Family history was also negative. On intraoral examination, two blood filled blisters were present on the middle of lateral border of the tongue on right side. The approximate size was $4 \times 5 \mathrm{~mm}$, which were smooth surfaces, deep purple in color with surrounding erythema (Fig. 1). On palpation they were soft to firm, non-tender, and bleed on manipulation. Left lateral border of the tongue appeared normal. Based on the history of overheated impression tray touching the right lateral border of the tongue, its sudden appearance and clinical appearance, diagnosis of angina bullosa hemorrhagica of right lateral border of the tongue was made. Differential diagnosis included other mucosal conditions such as erythema multiforme, bullous lichen planus, pemphigus, pemphigoid and epidermolysisbullosa.

\section{METHODS}

Methods of this case management started with routine blood an investigation including the

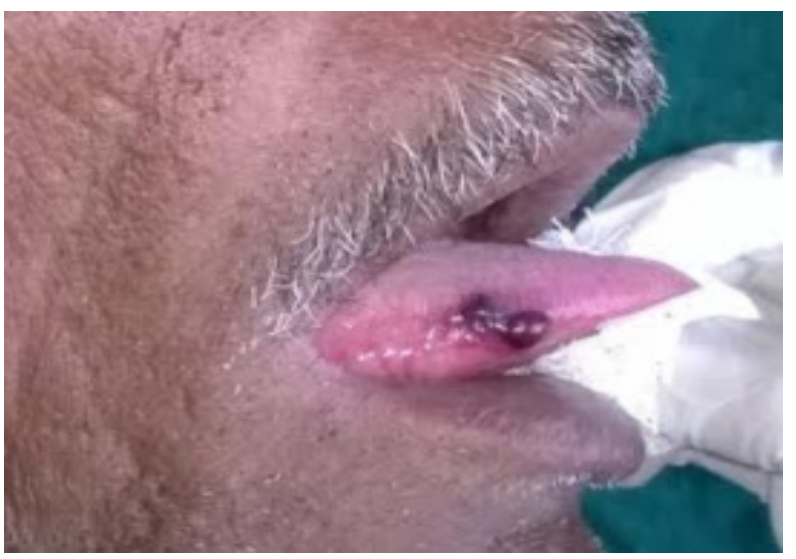

Figure 1. Photograph of the patient showing blood filled bullae on the right lateral border of the tongue

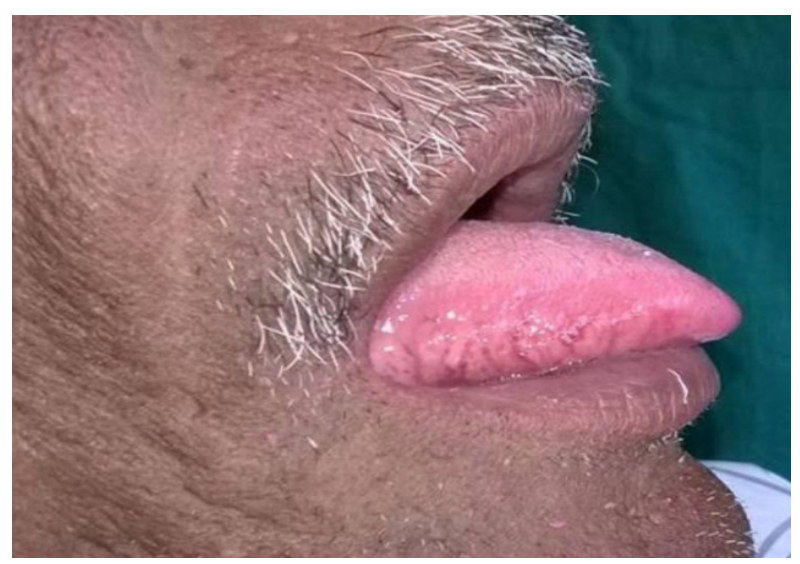

Figure 2. Intraoral photograph of the patient after one week 
coagulation profile that was within normal limits. In treatment, patient was advised to avoid hot and rough food items. Topical application chlorhexidine gel $(1 \%)$ and lignocaine gel $(2 \%)$ was prescribed for local application 4-5 times in a day on the lesion. Patient was reviewed after one week. Written informed consent was obtained from the patient for publication of this case report and accompanying images.

\section{RESULT}

After one week therapy with the lesion was healed (Fig. 2) and patient was referred back to Department of Prosthodontics for further management of edentulous mouth With very careful handling to prevent recurrence.

\section{DISCUSSION}

$\mathrm{ABH}$ has been traditionally described as a relatively rare condition. But, Slezak and Yamamoto et al. ${ }^{6,7}$ suggested that $A B H$ has been considered to be more common as ABH is mostly asymptomatic and hence is not perceived by the patient, thus not diagnosed. ABH mostly affects the soft palate, but can also occur on buccal mucosa, lip, and the lateral surface of the tongue; masticatory mucosa of the hard palate and gingiva does not seem to be affected. ABH mostly occur in middleaged and elderly people, with no apparent gender predilection. In the present case, the patient was male of middle age, and the site of involvement was lateral border of tongue, uncommon site to be involved.

The exact etiology of $A B H$ is not clear. However, some predisposing factors such as steroid-based inhalers and Diabetes mellitus are documented. ${ }^{6}$ The long standing use of inhaled steroids is known to affect collagen synthesis, with subsequent atrophy of the mucous epithelium. Also the tissue elasticity may decrease with the maturation of these collagen fibers, leading to poor support provided by blood vessels and hence the development of $A B H$ occurs even in response to minor trauma. ${ }^{8}$ Garlic et al. ${ }^{9}$ reported that excessive mechanical forces during mastication or minor thermal injuries are possible causes of $\mathrm{ABH}$. Also during mastication, the blood flow in the soft palate significantly increases via parasympathetic reflex vasodilatation. ${ }^{6}$ Other authors have suggested that blisters could be the result of a weakened junction between epithelial and connective tissue, making non-keratinized mucosa more susceptible to trauma. ${ }^{10}$

$A B H$ usually occurs at the junction between the hard and soft palate. ${ }^{4} \mathrm{ABH}$ lesions have a sudden onset, during or immediately after meals, and are preceded by a burning sensation and feeling of dry mouth. Blisters are generally painless and rupture spontaneously within a few hours from their appearance, releasing red blood contents and producing erosion areas that heal within 7 to 10 days. $^{7}$ Blisters affecting the soft palate and uvula were associated with pain and burning sensation after rupture, differently from patients with blisters on the tongue, which were totally asymptomatic.

In the present case, the blisters were asymptomatic, small and did not affect patients' dietary and respiratory functions. The etiology in the present case could be attributed to trauma during prosthodontics impression making. Blisters usually reach $2-3 \mathrm{~cm}$ in diameter and burst spontaneously, leaving ragged ulcers that heal without scarring but the lesions can recur. ${ }^{11}$

The diagnosis of $A B H$ is essentially based on clinical data. It has been reported previously that on histopathological examination, there is presence of nonspecific ulcers containing a chronic inflammatory infiltrate consisting of lymphocytes mainly. ${ }^{12}$ Immunoflorescence studies demonstrate no evidence of $\lg G$, IgM, IgA or C3 antibodies within the epithelium or basement membrane zone. ${ }^{12} \mathrm{ABH}$ can be easily confused with those occurring in many dermatological and systemic disorders. If there is a typical history of rapid blistering disease with absence of any dermatological, hematological or systemic sign and normal healing of the ulcers generally lead to the diagnosis of $\mathrm{ABH}$.

Patients with bleeding disorders (thrombocytopenia and von Willebrand's disease) can present with intra-oral blood filled lesions but bleeding and clotting test will distinguish these conditions. ${ }^{13}$ The absence of lesions on gingiva and nasal or conjunctival mucosa will differentiate it from benign mucous membrane pemphigoid. Linear IgA disease and dermatitis 
herpetiformis usually can be differentiated by the presence of a pruritic rash. In oral bullous lichen planus, bullae are generally associated with white striated pattern. The target or bull like lesion of the skin in erythema multiforme helps to distinguish it. The hemorrhagic bullae found in amylodosis are usually persistent and other clinical features of the disease like macroglossia and petechiae are present. Epidermolysis bullosa can be differentiated by the presence of bullous skin lesions. ${ }^{14}$

Thus, the previous medical and dental history of these patients should be thoroughly revised to help differentiating between $A B H$ and other systemic disorders. The assessment of hematologic and coagulation disorders should be performed to exclude the possibility of blood dyscrasia. Some authors have reported association between $\mathrm{ABH}$ and systemic disorders such as hypertension and diabetes, but there is need of further studies to confirm any such correlation. ${ }^{5}$

The management of a patient presenting with oral blood-filled bulla should include the detailed medical history and through clinical examination to differentiate $A B H$ from other systemic conditions. If there is any suspicion about the nature of the lesion, then biopsy and direct immunofluorescence should be performed to exclude more serious diseases. Routine hemogram including coagulation tests should always be performed to exclude blood dyscrasias. In the present case, all the blood investigations including coagulation profile was not altered, which helped in ruling out other blood dyscrasias.

Large palatal or pharyngeal blister causing a choking sensation should be surgically treated if still intact. $A B H$ treatment may include the use of anti-inflammatory and antibiotic agents, as well as antiseptic mouthwash containing $0.25 \%$ or $0.12 \%$ chlorhexidine digluconate, to help relief painful symptoms and avoid secondary infections. ${ }^{3}$ Long term follow up is recommended to exclude other serious conditions with similar clinical appearance. In the present case, as the condition was asymptomatic and etiology suspected was trauma from impression making procedure, so the management included topical antimicrobial such as chlorhexidine gel (1\%) to prevent secondary infection and topical anaesthetic such as lignocaine gel (2\%), to avoid discomfort while eating.

Surgical drainage is not necessary in small, localized lesions that do not functionally affect the airways, as these lesions are likely to rupture within some hours. Conversely, it is indicated whenever large blisters are present in the palate, causing a feeling of suffocation. ${ }^{15}$

Once again, the diagnosis for $A B H$ is difficult because asymptomatic and heals spontaneously without scarring and its rare appearance. The diagnosis of the lesion is very important as a rapidly expanding blood-filled bulla in the oropharynx can cause upper airway obstruction. Therefore, a high level of suspicion is warranted on part of dentists who may the first to encounter the lesion. Regardless of its typical clinical signs and course it probably often remains undiagnosed. Detailed anamnestic data gathering represents an inseparable part of the examination. It helps to avoid abundant diagnostic and therapeutic procedures, which may unnecessarily strain the patients.

\section{CONCLUSION}

Spontaneous Angina bullosa hemorrhagica of tongue was an unusual problem that can occur following prosthodontic procedure. The management of the lession were intended to reduce pain or discomfort and prevent secondary infections.

\section{REFERENCES}

1. Badham NJ. Blood blisters and the esophageal cast. J Laryngol Otol 1967;81(7):791-803.

2. Singh D, Agrawal S, Shashikanth MC, Deepak U3, Mehra A. Angina bullosa haemorrhagica. BMJ Case Report 2013; doi:10.1136/bcr-2012008505.

3. Gonçalves LM, Bezerra Júnior JR, Cruz MC. Clinical evaluation of oral lesions associated with dermatologic diseases. An Bras Dermatol 2010;85:150-6.

4. Giuliani M, Favia GF, Lajolo C, Miani CM. Angina bullosa haemorrhagica: presentation of eight new cases and a review of the literature. Oral Dis 2002;8(1):54-8.

5. Horie N, Kawano R, Inaba J, Numa T, Kato T, Nasu $D$ et al. Angina bullosa hemorrhagica of 
the soft palate: a clinical study of 16 cases. J Oral Sci 2008Mar;50(1):33-6.

6. Yamamoto $K$, Fujimoto $M$, Inoue $M$, Maeda M, Yakawa N, Kirita T. Angina bullosa hemorrhagica of the soft palate: report of 11 cases and literature review. J Oral Maxillofac Surg 2006;64(9):1433-6.

7. Slezák R. Traumatic haemorrhagic bullae of the oral mucosa (angina bullosa haemorrhagica). Folia Gastroenterol Hepatol 2005;3(4):122-7.

8. Shoor H, Mutalik S, Pai KM. Angina bullosa haemorrhagica. BMJ Case Rep 2013. p. 1-3. Narang T, Kanwar AJ. Hemorrhagic blisters in the mouth. Indian J Dermatol Venereol Leprol 2008;74:431.

9. Garlick JA, Calderon S. Oral blood blisters in angina bullosa haemorrhagica secondary to trauma of eating and dental injection. Br Dent J 1988:165(8);286-7.

10. Martini MZ, Lemos CA, Jr. Shinohara EH.
Angina bullosa hemorrhagica: report of 4 cases. Minerva Stomatol 2010;59(3):139-42.

11. Pahl C, Yarrow S, Steventon N, Saeed NR, Dyar O. Angina bullosa haemorrhagica presenting as acute upper airway obstruction. $\mathrm{Br}$ J Anaesth 2004;92:283-6.

12. Narang T, Kanwar AJ. Hemorrhagic blisters in the mouth. Indian J Dermatol Venereol Leprol 2008;74:431.

13. Curran AE, Rives RW. Angina bullosa haemorrhagica: an unusual problem following periodontal therapy. J Periodontol 2000;71:1770-3.

14. Neville BW, Damm DD, Allen CM, Bouquot JE. Oral \& Maxillofacial Pathology. $3^{\text {rd }}$ ed. St. Louis, Mo: Saunders Elsiever; 2009. p. 776.

15. Serra D, De Oliveira HS, Reis JP, Vieira R, Figueiredo A. Angina bullosa haemorrhagica: a disorder to keep in mind. Eur J Dermatol 2010;20(4):509-10. 